\title{
Mortalität von Patientinnen und Patienten auf einer Abteilung für Alterspsychiatrie in Graz, Österreich - Eine retrospektive Fall-Kontroll-Studie
}

\author{
Éva Rásky · Erwin Stolz · Christian Jagsch
}

Eingegangen: 27. September 2019 / Angenommen: 16. Dezember 2019 / Online publiziert: 22. Januar 2020

(c) Der/die Autor(en) 2020

\section{Zusammenfassung}

Grundlagen Trotz der demografischen Entwicklung und der steigenden Zahl der psychiatrischen Erkrankungen älterer Menschen gibt es nur wenige wissenschaftliche Untersuchungen zu den Merkmalen von Patientinnen und Patienten in der Alterspsychiatrie und zu deren Sterblichkeit. In dieser retrospektiven Fall-Kontroll-Studie werden Patientendaten untersucht, um festzustellen, in welchen Merkmalen sich die dort verstorbenen von den entlassenen Patientinnen und Patienten unterscheiden.

Methodik Insgesamt wurden 284 Patientenakten der Abteilung für Alterspsychiatrie und Alterspsychotherapie des Landeskrankenhaus Graz II ausgewertet. Zwischen 01.01.2015-31.12.2017 verstarben 51 Frauen und 91 Männer (= Fälle) im Krankenhaus. Diese wurden mit 142 nach Geschlecht gematchten und im selben Zeitraum entlassenen Patientinnen und Patienten (= Kontrollgruppe) verglichen. Die Auswertung erfolgte mittels logistischer Regressionsmodelle.

Ergebnisse Die auf der alterspsychiatrischen Station verstorbenen Patientinnen und Patienten waren deutlich älter und bei deutlich schlechterer physischer Gesundheit als die entlassenen Patientinnen und Patienten. Die Verstorbenen hatten gegenüber den Entlassenen eine 2,7-fach erhöhte Chance für eine ischämische Herzkrankheit, eine 2,5- bzw. 3-fach erhöhte Chance aus einem Pflegeheim bzw. aus einem Krankenhaus überwiesen worden zu sein sowie eine 3-fach erhöhte Chance für einen geringen funk-

\author{
É. Rásky $(\bowtie) \cdot$ E. Stolz \\ Institut für Sozialmedizin und Epidemiologie, Medizinische \\ Universität Graz, Graz, Österreich \\ eva.rasky@medunigraz.at \\ C. Jagsch \\ Abteilung für Alterspsychiatrie und Alterspsychotherapie, \\ LKH Graz II, Graz, Österreich
}

tionellen Status. Die Verstorbenen hatten zudem eine 4-fach erhöhte Chance hoher CRP-Werte und einer Delir Diagnose gegenüber den Entlassenen.

Schlussfolgerungen Die verstorbenen Patientinnen und Patienten hatten einen erheblich schlechteren Gesundheitszustand und waren eher delirant verglichen mit den entlassenen Patientinnen und Patienten. Strukturell müssten gesundheitspolitische Vorkehrungen getroffen werden, damit nicht unnötige Transfers in alterspsychiatrische Abteilungen notwendig werden.

Schlüsselwörter Gerontopsychiatrische Patienten/ Patientinnen, stationär · Sterblichkeit · Prädiktoren

\section{Mortality of gerontopsychiatric inpatients in Graz, Austria-a retrospective case-control study}

\begin{abstract}
Summary
Background Despite the demographic development and the increasing number of psychiatric diseases in older people, there are only few scientific investigations on the characteristics of patients in gerontopsychiatry and their mortality. In this retrospective case control study patient data were examined in order to establish which features of patients who died in a department of gerontopsychiatry show differences to those of patients who were discharged.

Method A total of 284 patient files from the department of gerontopsychiatry and geriatric psychotherapy of the State Hospital Graz II were evaluated. Between 1 January 2015 and 31 December 2017, a total of 51 women and 91 men died (= cases) in hospital. These patients were compared with 142 sex-matched patients (control group) who were discharged during the same period of time. The evaluation was carried out using logistic regression models.
\end{abstract}


Tab. 1 Unterschiede zwischen verstorbenen und nichtverstorbenen Patientinnen und Patienten

\begin{tabular}{|c|c|c|c|}
\hline \multirow{3}{*}{ Variablen } & \multicolumn{2}{|c|}{ Deskriptive Statistik } & \multirow{2}{*}{$\begin{array}{l}\text { Logistisches Regressi- } \\
\text { onsmodell }\end{array}$} \\
\hline & $\begin{array}{l}\text { Verstorbene } \\
(n=142)\end{array}$ & $\begin{array}{l}\text { Nicht-Verstorbene } \\
(n=142)\end{array}$ & \\
\hline & $\mathrm{MW}(\mathrm{SD}) / n(\%)$ & $\mathrm{MW}(\mathrm{SD}) / n(\%)$ & OR (95\%-KI) \\
\hline Alter (Jahre) & $84,0(7,8)$ & $77,7(8,6)$ & $3,3(1,5-7,1)$ \\
\hline Aufenthaltsdauer (Tage) & $14,3(20,5)$ & $19,1(16,7)$ & $1,1(0,6-2,1)$ \\
\hline \multicolumn{4}{|l|}{ Psychiatrische Diagnose } \\
\hline Delir (F05) & $88(78,6)$ & $24(21,4)$ & $4,0(1,8-8,7)$ \\
\hline Demenz (F00-F02.8) & $83(55,0)$ & $68(45,0)$ & $0,5(0,2-1,1)$ \\
\hline Depression (F32-F33) & $33(31)$, & $71(68,3)$ & $0,9(0,4-1,9)$ \\
\hline \multicolumn{4}{|l|}{ Somatische Diagnosen } \\
\hline Ischämische Herzkrankheiten (I20-I25) & $85(69,1)$ & $38(30,9)$ & $2,7(1,4-5,2)$ \\
\hline Zerebrovaskuläre Erkrankung (160-I69) & $32(50,0)$ & $32(50,0)$ & $0,7(0,3-1,5)$ \\
\hline Diabetes (E10-E11) & $36(62,1)$ & $22(37,9)$ & $1,8(0,8-4,2)$ \\
\hline Chronische Nierenkrankheit (N18) & $74(66,1)$ & $38(33,9)$ & $1,6(08,-3,2)$ \\
\hline Chronische Atemwegskrankheit (J00-J99) & $62(63,3)$ & $36(36,7)$ & $0,7(0,3-1,5)$ \\
\hline Erythrozyten/Ery (Mio/ml) & $4,1(0,7)$ & $4,4(0,6)$ & $1,4(0,7-2,8)$ \\
\hline C-reaktives Protein/CRP (g/ml) & $46,6(62,9)$ & $13,5(22,5)$ & $4,4(1,6-12,3)$ \\
\hline Schlechter Ernährungszustand & $55(66,3)$ & $28(33,7)$ & $1,5(0,7-3,0)$ \\
\hline Braden-Skala & $14,8(3,6)$ & $18,6(3,6)$ & $0,3(0,1-0,7)$ \\
\hline Vorheriger Aufenthaltsort: Privathaushalt & $32(27,8)$ & $83(72,2)$ & = Referenzkategorie \\
\hline Vorheriger Aufenthaltsort: Pflegeheim & $38(61,3)$ & $24(38,7)$ & $2,5(1,1-5,9)$ \\
\hline Vorheriger Aufenthaltsort: Krankenhaus & $71(67,6)$ & $34(32,4)$ & $3,0(1,3-6,8)$ \\
\hline
\end{tabular}

Results Patients who died on the geriatric psychiatry ward were clearly older and were in a clearly poorer physical state of health than the discharged patients. The deceased patients had a 2.7-fold higher chance of an ischemic cardiac disease as well as a 2.5-fold and 3.5-fold higher chance of being referred from a nursing home or hospital, respectively. They also had a threefold higher chance for a lower functional status, a fourfold increased chance of higher C-reactive protein (CRP) values and a diagnosis of delirium compared to discharged patients.

Conclusion The deceased patients had a substantially poorer state of health and tended to suffer more from delirium compared to the discharged patients. Structural health policy precautions must be undertaken so that unnecessary transfers to gerontopsychiatry departments do not become necessary.

Keywords Gerontopsychiatric inpatients · Mortality · Predictors

\section{Hintergrund und Fragestellung}

Die demografischen Entwicklungen führen zu Unterschieden in den Krankheitshäufigkeiten, nicht zuletzt zu vermehrten neurologischen und psychiatrischen Erkrankungen im Alter. Die Kenntnis der Prädiktoren für die Sterblichkeit der Patientinnen und Patienten dieser Krankheitsgruppen kann dazu beitragen, Qualitätsverbesserungen in der Versorgung und spezifische Präventionsmaßnahmen zu entwickeln. Mortalitätsursachen und Risikofaktoren für die Sterblichkeit sind daher wichtige epidemiologische Informationsquellen zur Vermeidung von Todesfällen in Bevölkerungen. Die Spitalssterblichkeit von älteren Patientinnen und Patienten nach Krankheitsgruppen kann entscheidende Hinweise für Qualitätsverbesserungen und Präventionsmaßnahmen in den Krankenhäusern liefern [1-3]. In den letzten Jahrzehnten wurden nur wenige Arbeiten spezifisch zur Sterblichkeit von geronto-psychiatrischen Krankenhauspatienten publiziert. Arbeiten aus den 1990er Jahren geben für diese Gruppen Mortalitätsraten zwischen 1-16\% an [4-6]. Insgesamt sind wissenschaftliche Studien zur Zahl und zu den Merkmalen der Verstorbenen dieser Alters- und Krankheitsgruppe im deutschsprachigen Raum rar. Eine systematische Übersichtsarbeit nennt für die Mortalität älterer Patientinnen und Patienten folgende Prädiktoren: Funktionalität, Schwere der Erkrankung, kognitive Einbußen, Komorbiditäten, aktuelle Erkrankung, Polypharmazie, Alter und Geschlecht [1]. Für stationäre alterspsychiatrische Patientinnen und Patienten werden in den wenigen internationalen Studien als Sterbeursache häufig Demenz und Depression sowie bereits mehrfache stationäre Aufenthalte angeführt [3, 4]. Als Haupttodesursachen 
werden Herz-Kreislauferkrankungen genannt [6, 7]. Die Suizidmortalität ist in der Spitalspopulation im Gegensatz zu dieser Altersgruppe in der Allgemeinbevölkerung mit sehr hoher Selbstmordrate eher gering $[4,8,9]$.

Ziel dieser retrospektiven Fall-Kontroll-Studie ist es, herauszufinden, welche Merkmale die verstorbenen gegenüber den entlassenen Patientinnen und Patienten der Abteilung für Alterspsychiatrie und Alterspsychotherapie des Landeskrankenhaus Graz II (vormals Süd-West) aufweisen und worin sich diese beiden Gruppen unterscheiden.

\section{Daten und Methoden}

\section{Daten}

Ausgewertet wurden 284 Patientenakten der Abteilung für Alterspsychiatrie und Alterspsychotherapie des Landeskrankenhaus Graz II (vormals Süd-West). Diese Abteilung mit 109 Betten und etwa 1800 stationären Aufnahmen pro Jahr betreut ein Einzugsgebiet von 1,2 Mio. Einwohnerinnen und Einwohnern. Damit hat die Abteilung eine wichtige Versorgungsfunktion. Denn für das Jahr 2017 weist die Statistik Austria 967 Betten für psychische Erkrankungen in allgemeinen Krankenanstalten aus und 3371 Spitalsentlassungen von über 65-Jährigen mit den ICD Kodes F00-F99 [10]. Zwischen 01.01.2015-31.12.2017 verstarben 51 Patientinnen und 91 Patienten (= Fälle) während ihres stationären Aufenthaltes. Sie wurden mit 142 nach Geschlecht gematchten und im selben Zeitraum entlassenen Patientinnen und Patienten (= Kontrollgruppe) verglichen. Die Daten wurden vor Ort aus den Patientenakten entnommen, geprüft, elektronisch kodiert und vor der Datenanalyse anonymisiert (Tab. 1).

Die zuständige Ethikkommission der Medizinischen Universität Graz hat die vorliegende Studie genehmigt (EK 29369 ex 16/17).

\section{Methoden}

Im Rahmen dieser Fall-Kontroll-Studie wurden verstorbene (Fälle) und entlassene Patienten und Patientinnen (Kontrollgruppe) deskriptiv und mittels multipler logistischer Regressionsanalyse verglichen, hinsichtlich ihres Alters, ihres Geschlechts, ihrer stationären Aufenthaltstage, psychiatrischer und somatischer Diagnosen, Biomarker (Erythrozyten/Ery und C-reaktives Protein/CRP), pflegerischem Assessment mittels Braden-Skala (misst sensorisches Empfindungsvermögen, Feuchtigkeit der die Haut ausgesetzt ist, Aktivität, Mobilität, Ernährung sowie Reibung und Scherkräfte) und Ernährungszustand sowie Aufenthaltsort vor Aufnahme (Privathaushalt, Pflegeheim oder Krankenhaus). Die Erhebung der Patientenmerkmale bezog sich jeweils auf den letzten verfügbaren Wert während des stationären Aufenthaltes mit Ausnahme der Braden-Skala, hier wurde der Wert zu
Beginn des Aufenthalts kodiert. Alle metrischen Prädiktoren wie Alter, Aufenthaltstage, Braden-Skala, Ery, CRP wurden mittelwertszentriert und durch zwei Standardabweichungen dividiert, um eine Vergleichbarkeit der Effekte mit den kategorialen Prädiktoren zu gewährleisten. Alle Berechnungen wurden mit der Statistikumgebung R v3.6.1 durchgeführt [11]. Für das Regressionsmodell wurden die in den Patientenakten fehlende Angaben (7,7\% Braden-Skala, 4,5\% Ernährungszustand, $2 \% \mathrm{CRP},>1 \%$ Erys und vorheriger Aufenthaltsort), insgesamt $12,3 \%$, mehrfach imputiert $(\mathrm{m}=10)$.

\section{Ergebnisse}

Die Mortalitätsrate (2015-2017) auf der Abteilung für Alterspsychiatrie und Alterspsychotherapie des Landeskrankenhaus Graz II (vormals Süd-West) betrug 4,2\% (142/3395). Die durchschnittliche Aufenthaltsdauer betrug 14,3 Tage (Median=11, Standardabweichung $=20,5$ ) bei den verstorbenen und 19,1 Tage (Median $=15$, Standardabweichung $=16,7$ ) bei den entlassenen Patientinnen und Patienten. Die Patientencharakteristika nach Mortalitätsstatus und die Ergebnisse der logistischen Regression sind in Tab. $1 \mathrm{zu}$ ersehen. Das logistische Regressionsmodell zeigt, dass die Patientinnen und Patienten, die auf der alterspsychiatrischen Station verstorben sind, erwartungsgemäß deutlich älter als auch bei deutlich schlechterer physischer Gesundheit waren als die entlassenen Patientinnen und Patienten. Im Vergleich zu den entlassenen, hatten die verstorbenen Patientinnen und Patienten eine 2,7-fach erhöhte Odds ratio/OR an einer ischämischen Herzkrankheit (I20-I25) zu leiden, hatten eine 2,5- bzw. 3-fach erhöhte OR aus einem Pflegeheim bzw. aus einem Krankenhaus in die Alterspsychiatrie überwiesen worden zu sein sowie eine 3-fach erhöhte OR für einen geringen funktionellen Status (d.h. einem niedrigen Wert auf der Braden-Skala). Zudem zeigten die Verstorbenen eine 4-fach erhöhte OR hoher CRP-Werte und einer Delir-Diagnose.

\section{Schlussfolgerungen und Diskussion}

Wie Hewer et al. [7] und Hwang et al. [6] haben auch wir in unserer Fall-Kontroll-Studie, in der wir in der Abteilung verstorbene und entlassene Patientinnen und Patienten in vorher festgelegten Merkmalen miteinander verglichen, nachweisen können, dass ischämische bzw. Herzkreislauferkrankungen bei den geronto-psychiatrischen Patientinnen und Patienten, wie auch in der Allgemeinbevölkerung in Ländern mit hohem Einkommen wie Österreich, die bedeutendste Todesursache darstellen [12]. Mit Ausnahme des Delirs als komplexem physiologischen Geschehen von Aufmerksamkeits- und Kognitionsdefiziten sind bei den geronto-psychiatrischen Patientinnen und Patienten Herzerkrankungen, eine somatische Erkrankung, als Sterbeursache bedeutend [13]. Zentrale 
Merkmale waren neben Delir und Herzkreislauferkrankungen, das Alter, die Höhe des CRP-Wertes und der Wert auf der Braden-Skala sowie der Ort von dem die Patientinnen und Patienten überwiesen wurden. Auch Untersuchungen in allgemeinmedizinischen Abteilungen weisen auf den Risikofaktor Delir als Todesursache hin [14]. Eine systematische Literaturübersicht gibt folgende Variablen für einen schlechten Outcome an: Länge des Delirs, motorisch hypoaktiver Subtyp, Schwere des Delirs, vorbestehende psychiatrische Erkrankungen wie Demenz und Depression [14]. In der Literatur gibt es Hinweise, dass Spitalspatienten ohne Demenz, die ein Delir entwickeln, schwerer erkrankt sind als diejenigen mit vorbestehenden Hirnpathologien [15]. Allerdings zeigten van Roessel et al. [16], dass Studien keine Differenz in der Mortalität nach Delir bei dementen und nicht dementen Patientinnen und Patienten nachweisen konnten. Auch in unserer Studie zeigte sich kein Interaktionseffekt zwischen Demenz und Delir. Die Höhe des Entzündungsparameters CRP war in unserem Patientenkollektiv ein bedeutender biologischer Marker im Zusammenhang mit der Sterblichkeit. Eher sozial zu wertende Prädiktoren wie schlechter Ernährungszustand und das Krankenhaus oder ein Pflegeheim als vorherigem Aufenthaltsort stellten ein gewisses Sterberisiko dar.

Nachdem ein Delir das Risiko zu sterben für ältere Patientinnen und Patienten erhöht, wäre es sinnvoll, bereits auf inneren und chirurgischen Abteilungen, aber auch in Langzeiteinrichtungen geriatrische Assessments durchzuführen, um das spezifische Risiko für die einzelne Patientin, den einzelnen Patienten abzuklären und entsprechende Maßnahmen zu setzen, um die Sterblichkeit geronto-psychiatrischer Patientinnen und Patienten zu senken [17-20]. Strukturell müssten gesundheitspolitische Vorkehrungen getroffen werden, dass für die verhaltensauffälligen/ deliranten sterbenden Patientinnen und Patienten in den Krankenhäusern und Langzeiteinrichtungen eine Konsiliar- oder Liaison-Alterspsychiatrie zur Verfügung steht oder die Betroffenen im Bedarfsfall palliativ versorgt werden können, damit nicht unnötige Transfers in alterspsychiatrische Abteilungen notwendig werden. In unserer Studie wurde ein bedeutender Anteil von Patientinnen und Patienten aus Krankenhäusern in die Alterspsychiatrie überwiesen (Tab. 1).

Grundsätzlich lässt sich festhalten, dass die Einflussfaktoren für die Behandlungsergebnisse von älteren Spitalspatienten komplex sind. In einer Übersichtsarbeit wurden Outcomes wie Aufenthaltsdauer, Sterblichkeit, Entlassungsdestination, Wiederaufnahmezahl untersucht [1]. In unserer Studie wählten wir als Outcome die Mortalität. Nicht alle in der Übersichtsarbeit von Campbell u.a. [1] genannten Parameter konnten wir aus den Krankenakten extrahieren. Beispielsweise waren die Angaben zur Medikamenteneinnahme in den Arztbriefen durch die ungleiche Verteilung der fehlenden Angaben zwischen den Verstorbenen und Entlassenen für die Analyse nicht valide zu verwerten. Um den Outcome bei älteren Patientinnen und Patienten gut bestimmen $\mathrm{zu}$ können, müssten aber neben Routinedaten wie Alter, Geschlecht und Erkrankung auch facettenreiche Aspekte wie der funktionelle Status und die Kognition erfasst werden. Weitere Limitationen unserer Studie umfassen die fehlenden Informationen zur Schwere der Erkrankung, der Kognition (Mini Mental State Examination/MMSE) - herzu gab es bei 103 der 142 verstorbenen und bei 43 der 142 entlassenen Patientinnen und Patienten keine Angabe in den Krankenakten -, und die Anzahl stationärer Voraufenthalte, die wir nicht erhoben haben. Zudem zeigt sich aufgrund der begrenzten Stichprobe $(n=284)$ eine bedeutsame Schwankungsbreite hinsichtlich der Effektstärke mehrerer wichtiger Einflussfaktoren. In dieser Fall-Kontroll-Studie wurde die Spitalssterblichkeit untersucht. Eine Aussage inwieweit die entlassenen Patientinnen und Patienten (die Kontrollgruppe) dann in der weiteren Folge gestorben sind, war daher nicht möglich.

Wichtig wäre es in Zukunft, die in der Literatur beschriebenen Merkmale auch in alterspsychiatrischen Abteilungen zu erheben, um auch für diese Patientengruppe Aussagen zu den komplexen Ursachen für die Sterblichkeit machen zu können, um Qualitätsverbesserungen in deren Behandlung einzuleiten oder entsprechende Präventionsmaßnahmen entwickeln zu können. Diese Daten stehen derzeit in Österreich nicht in ausreichendem Maße zur Verfügung.

Funding Open access funding provided by Medical University of Graz.

Interessenkonflikt É. Rásky, E. Stolz und C. Jagsch geben an, dass kein Interessenkonflikt besteht.

Open Access Dieser Artikel wird unter der Creative Commons Namensnennung 4.0 International Lizenz veröffentlicht, welche die Nutzung, Vervielfältigung, Bearbeitung, Verbreitung und Wiedergabe in jeglichem Medium und Format erlaubt, sofern Sie den/die ursprünglichen Autor(en) und die Quelle ordnungsgemäß nennen, einen Link zur Creative Commons Lizenz beifügen und angeben, ob Änderungen vorgenommen wurden.

Die in diesem Artikel enthaltenen Bilder und sonstiges Drittmaterial unterliegen ebenfalls der genannten Creative Commons Lizenz, sofern sich aus der Abbildungslegende nichts anderes ergibt. Sofern das betreffende Material nicht unter der genannten Creative Commons Lizenz steht und die betreffende Handlung nicht nach gesetzlichen Vorschriften erlaubt ist, ist für die oben aufgeführten Weiterverwendungen des Materials die Einwilligung des jeweiligen Rechteinhabers einzuholen.

Weitere Details zur Lizenz entnehmen Sie bitte der Lizenzinformation auf http://creativecommons.org/licenses/by/4. $0 /$ deed.de.

\section{Literatur}

1. Campbell SE, Seymour DG, Primrose WR, ACMEPLUS project. A systematic literature review of factors affecting out- 
come in older medical patients admitted to hospital. Age Ageing. 2004;33(2):110-5.

2. Freyne A, Wrigley M. Acute inpatient admissions in a community oriented old age psychiatry service. Ir J Psychol Med. 1997;14(1):4-7.

3. Ifteni P, Grudnikoff E, Koppel J, Kremen N, Correll CU, Kane JM, et al. Haloperidol and sudden cardiac death in dementia: autopsy findings in psychiatric inpatients. Int $\mathrm{J}$ Geriatr Psychiatry. 2015;30(2):1224-9.

4. BlackD, JolleyD. Slow euthanasia? The deaths of psychogeriatric patients. BMJ. 1990;300:1321-3.

5. Rockwood K, Stolee P, Brahim A. Outcomes of admission to a psychogeriatric service. Can J Psychiatry. 1991;36(4):275-9.

6. Hwang JP, Tsai SJ, Yang CH. Mortality in geriatric psychiatric inpatients. IntJ Psychiatry Med. 1998;28(3):327-31.

7. Hewer W, Rössler W, Falkenheuer B, Löffler W. Mortality among patients in psychiatric hospitals in Germany. Acta Psychiatr Scand. 1995;91:174-9.

8. Black D, Jolley D. Deaths in psychiatric care. Int J Geriatr Psychiatry. 1991;6(7):489-95.

9. Barbosa S, Sequeira M, Castro S, Manso R, Klut Câmara C, Trancas B, et al. Causes of death in an acute psychiatric inpatient unit of a Portuguese general hospital. Acta Med Port. 2016;29(7-8):468-75.

10. Statistik Austria. Jahrbuch der Gesundheitsstatistik 2017. Wien: StatistikAustria;2019. S. 273, 120.

11. Team RC. R: A language and environment for statistical computing. Version 3.6.1..Vienna:Team RC;2019.

12. World Health Organization. Global burden of diseases, part 2: causes of death. Geneva: WHO; 2008. S. 12. http:// origin. who.int/healthinfo/global_burden_disease/GBD_report_ 2004update_part2.pdf.
13. Hshieh TT, Inouye SK, Oh ES. Delirium in the elderly. Psychiatr Clin North Am. 2018;41(1):1-17.

14. JacksonTA, WilsonD, Richardson S, LordJM.Predicting outcome in older hospital patients with delirium: a systematic literature review. Int J Geriatr Psychiatry. 2016;31(4):392-9.

15. Hapca S, Guthrie B, Cvoro V, Bu F, Rutherford AC, Reynish E, et al. Mortality in people with dementia, delirium, and unspecified cognitive impairment in the general hospital: prospective cohort study of 6,724 patients with 2 years follow-up. Clin Epidemiol. 2018;10:1743-53.

16. Van Roessel S, Keijsers CJPW, Romijn MDM. Dementia as a predictor of morbidity and mortality in patients with delirium. Maturitas. 2019;125:63-9.

17. Britton A, Russell R. Multidisciplinary team interventions for delirium in patients with chronic cognitive impairment. Cochrane Database Syst Rev. 2001; https://doi.org/ 10.1002/14651858.CD000395.pub2.

18. Siddigi N, House AO, Holmes JD. Occurence and outcome of delirium in medical in-patients: a systematic literature review. AgeAgeing. 2006;35(4):350-64.

19. Clegg A, Siddiqi N, Heaven A, Young J, Holt R. Interventions for preventingdeliriumin older peopleininstitutionallongterm care. Cochrane Database Syst Rev. 2014; https://doi. org/10.1002/14651858.CD009537.pub2.

20. Eamer G, Taheri A, Chen SS, Daviduck Q, Chambers T, ShiX, et al. Comprehensive geriatric assessment for older people admitted to a surgical service. Cochrane Database Syst Rev. 2018; https:// doi.org/10.1002/14651858.CD012485.pub2.

Hinweis des Verlags Der Verlag bleibt in Hinblick auf geografische Zuordnungen und Gebietsbezeichnungen in veröffentlichten Karten und Institutsadressen neutral. 